\title{
Reduction of patient radiation dose during percutaneous CT vertebroplasty: Impact of a new computer-assisted navigation (CAN) system
}

\author{
C. Teriitehau ${ }^{1, *}$, H. Rabeh ${ }^{2}$, E. Pessis ${ }^{1}$, Q. Sénéchal ${ }^{1}$, F. Besse ${ }^{1}$ and M. Bravetti ${ }^{1}$ \\ ${ }^{1}$ Interventional Radiology Unit, Imaging Department, Centre Cardiologique du Nord, 32-36 Rue des Moulins Gémeaux, \\ 93200 Saint-Denis, France. \\ 2 IMACTIS, 20 Rue du Tour de l'Eau, 38400 Saint-Martin-d'Hères, France.
}

Received: 20 November 2019 / Accepted: 30 November 2019

\begin{abstract}
To assess the impact of a computer assisted navigation system (CAN) (CT-Navigation ${ }^{\mathrm{TM}}$ IMACTIS, France) on patient radiation doses during percutaneous CT vertebroplasty a retrospective comparative trial was performed and included 37 patients requiring percutaneous vertebroplasty. This study was approved by CCN (Centre Cardiologique du Nord, Saint-Denis, France) ethical committee; all patients provided informed consent. All procedures were conducted in the interventional radiology department at $\mathrm{CCN}$, by an experienced single radiologist using the same model and CT scan with identical parameters. The interventional dose length product (IDLP), representing the absorbed dose by the length of explored organs during the needle insertion phase, was compared in 15 consecutive patients who underwent a conventional procedure (CT control group), and in 22 patients who underwent CAN CT vertebroplasty (CAN group). The IDLP difference between the two groups was evaluated using Mann-Whitney U test. The median IDLP dose for the CAN group was $305.6 \mathrm{mGy} . \mathrm{cm}$ [182.3;565.4], representing a reduction by a 3.2 factor compared with that of the conventional CT group (median $975.2 \mathrm{mGy} . \mathrm{cm}[568.3 ; 1077.1] ; p<0.001$ ). The median procedure duration for the CAN group was $50 \min [35 ; 60]$ vs. $100 \mathrm{~min}[82 ; 100]$ in the CT group $(p<0.001)$, representing a $50 \%$ reduction. In experienced hands, use of a CT-Navigation ${ }^{\mathrm{TM}}$ system (IMACTIS ${ }^{8}$ ) significantly reduced both patient radiation dose and procedure duration when compared to conventional CT guided percutaneous vertebroplasty.
\end{abstract}

Keywords: interventional radiology / CT / vertebroplasty / navigation system / radiation dose reduction / procedure time reduction

\section{Introduction}

The number of spinal interventional radiology procedures has increased during the last decade due to rising spine-related disorders (Toma et al., 2007; Friedly et al., 2010). This trend has led to greater use of X-ray guidance, with higher radiation exposure for both patients and health professionals (Perisinakis et al., 2004). In parallel, there is greater awareness of concern about possible excessive X-ray exposure and associated risks (Synowitz and Kiwit, 2006; Li et al., 2013; Panizza et al., 2013; Narain et al., 2017). Thus, the International Commission on Radiological Protection(ICPR) has established the principles for radiation protection, i.e., justification and optimization for both patients and workers with the additional principle of dose

\footnotetext{
*Corresponding author: cteriitehau@me.com
}

limitation for the workers (the maximum equivalent dose limit for the ocular lens is 20 and $500 \mathrm{mSv}$ to the extremities). However, such dose limits may be exceeded in the operating room (Feghali et al., 2014), especially during percutaneous vertebroplasty because of potential repetition of scouting exposures due to a limited view of the surgical field (Komemushi et al., 2005; Synowitz and Kiwit, 2006). Thus both patients' and workers' dose reduction are necessary (Bernier et al., 2012; Bourguignon et al., 2017) to reduce the risk of cancer (Jacob et al., 2013).

Several researchers highlighted the ongoing unmet need for a solution to help reduce this radiation exposure (Synowitz and Kiwit, 2006; Lonjon et al., 2016). They also suggested that navigation systems developed to guide physicians during interventional procedures may have the potential to reduce the X-ray dose delivered (Izadpanah et al., 2009; Narain et al., 2017) to both patients and workers. 
The objective of this study conducted in the interventional radiology department at Centre Cardiologique du Nord (CCN) was to assess the impact of a navigation system (CTNavigation $^{\text {TM }}$ by IMACTIS ${ }^{\circledR}$, France) on radiation doses delivered to patients during percutaneous CT vertebroplasty, testing the hypothesis that using this navigation system may reduce the interventional dose length product (IDLP) during the insertion phase of a vertebroplasty procedure.

\section{Materials and methods}

\subsection{Study design}

This retrospective open comparative controlled, single center-single radiologist study was approved by the $\mathrm{CCN}$ ethics committee.

Thirty-seven sequential patients, who underwent a percutaneous CT vertebroplasty procedure from October 2016 to February 2017, were included in the interventional radiology department at $\mathrm{CCN}$, where a computer assisted navigation system (CAN) has been used for vertebroplasty since December 2016. The first 21 consecutive patients treated with CAN vertebroplasty were compared to the last 16 patients treated with a conventional vertebroplasty CT procedure.

\subsection{Interventions}

One CT interventional radiologist with a 20 year-experience (about 700 conventional procedures performed) carried out all interventions. The operator was first trained to use the CAN system by performing two interventions on a phantom model. All procedures were performed using a Somatom, AS +128 (Siemens, Erlangen, Germany) CT scanner using the same parameters for all interventions, e.g., $2.4 \mathrm{~mm}$ slice thickness, pitch of 1 .

\subsubsection{CT control group}

For the CT control group, the intervention was performed according to the conventional procedure: the patient was placed prone on the operation table. The entry point was defined under CT guidance. Local anesthesia or general anesthesia were used according to the clinical needs. A 13 gauge (and/or) 11-gauge needle (Osteo-Site ${ }^{\circledR}$ Cook medical) were inserted at the entry site until the tip reached the center of vertebral body under intermittent CT fluoroscopy monitoring. This needle insertion was performed under sequential acquisition (a simple 4-cm CT volume exploration in every acquisition). The use of this sequential mode allowed the placement of the needle without tilting the gantry. Then, after verifying the position of the needle a commercially available polymethyl methacrylate (PMMA) cement (Biomet bone cement, Zimmer Biomet) was carefully injected into the treated vertebral body under intermittent monitoring in the anteroposterior or lateral projection to ensure adequate filling of the lesion under fluoroscopic guidance(Orbic, Siemens, Erlangen, Germany).

\subsubsection{CAN group}

For the CAN group, the procedure was performed using the aforementioned CT scanner and was assisted by the navigation system (CT-Navigation $^{\text {TM }}$, IMACTIS ${ }^{\circledR}$, France).
CT-Navigation allows electromagnetic navigation based on CT-image guidance and consists of three components: a field generator integrated in a patient referential (PR) taped on patient skin, a magnetic receiver integrated in a needle holder, and a central control unit with the appropriate data acquisition system and navigation software (Fig. 1).

The generated magnetic fields are used in the human body, in a similar manner to a GPS navigation system. The magnetic field generator integrated in the patient referential (analogous to a satellite in space) is placed on the patient (within $20 \mathrm{~cm}$ maximum from the lesion) and create a magnetic field centered around the lesion of interest. The electromagnetic receiver (analogous to a car) integrated in the needle holder can then be localized within the field. The position and the orientation of the needle holder are tracked and thus the anticipated needle trajectory is displayed on a monitor. Once the patient referential (PR) was fixed on the patient and sterile preparations were performed, a CT acquisition including the PR was conducted and then transferred to the navigation station. The interventional radiologist can insert the 11 or 13 needle gauge and compare the real time anticipated needle position and the planned trajectory displayed on the same screen (Fig. 1). During needle insertion, additional CT acquisition could be performed and transferred to the navigation station to check the progression of the needle tip. As soon as the needle reached the planned distal point of the trajectory, polymethyl methacrylate cement is injected carefully as in the conventional procedure.

\subsection{Outcomes}

The primary study end point was to measure the patient radiation dose delivered during needle insertion defined as the interventional Dose length product (IDLP), i.e., the interventional dose length product which represents the absorbed dose by the length of the explored organs during the needle insertion phase.

Secondary outcomes included the following:

- the total dose length product defined as the absorbed dose by the length of explored organs during the total duration of the intervention;

- the procedure duration defined as the time elapsed between the first and the final CT acquisitions; - complications.

\subsubsection{Statistical methods}

Descriptive statistics were used for the presentation of patients' characteristics and outcomes data. Continuous variables were expressed as mean and standard deviation or medians and range where appropriate. Mann-Whitney $U$ test was used to evaluate the possible difference between groups. Categorical variables were compared using the Chi-square test or the Fisher exact test. The threshold for statistical significance was set to $p \leq 0.05$. All analyses were performed using statistical software $\mathrm{R}$ v3.5.3 (R Core Team, R foundation).

\section{Results}

\subsection{Baseline data}

A total of eighty-eight vertebrae were treated in the thirty-seven patients enrolled between October 2016 and 

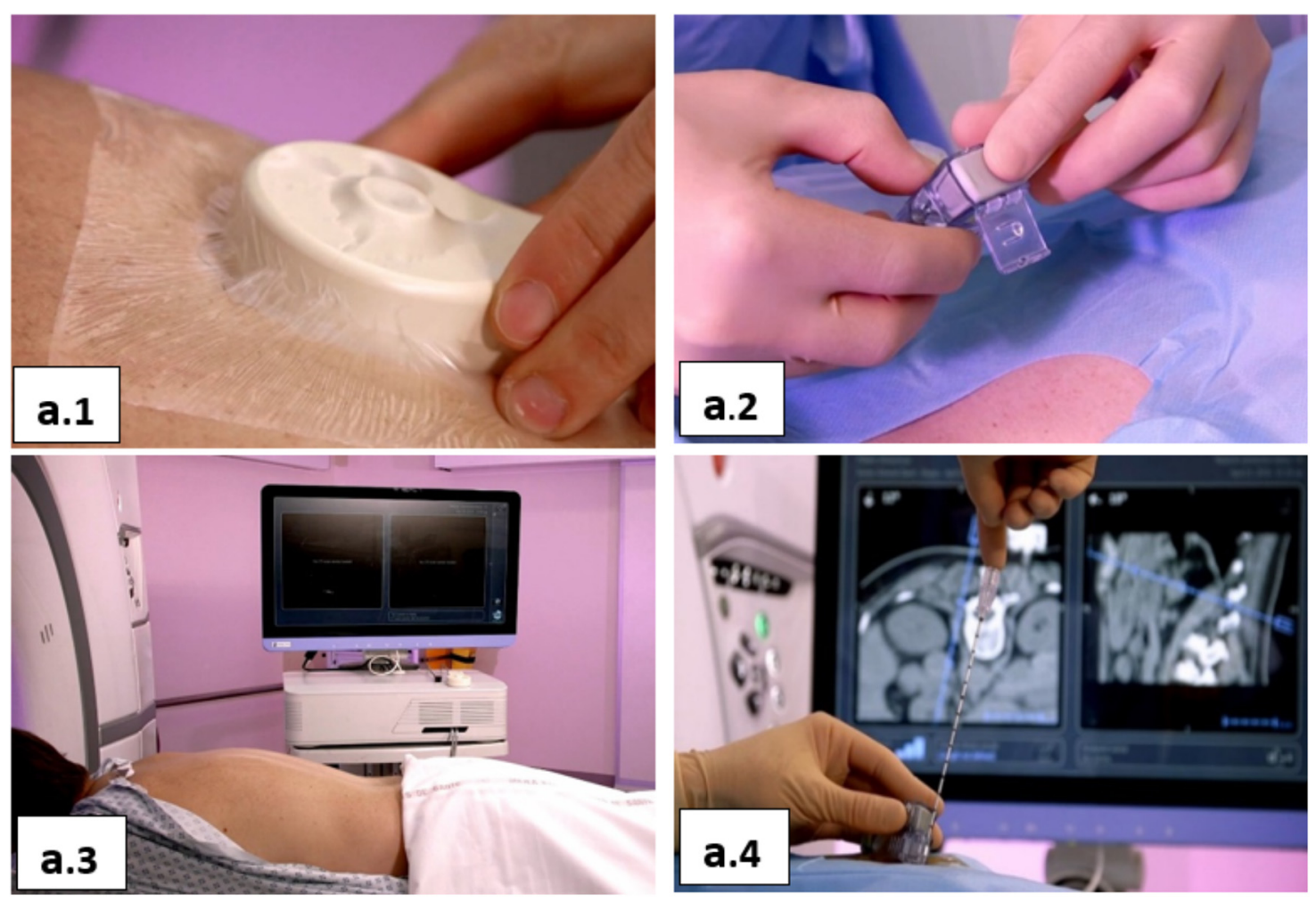

Fig. 1. Computer-assisted electromagnetic navigation system (CT-Navigation, IMACTIS ${ }^{\circledast}$, France). a.1: field generator integrated in the patient referential; a.2: magnetic receiver integrated in a needle holder; a.3: central control unit with the appropriate data acquisition system and navigation software; a.4: needle insertion while comparing real time anticipated needle position with planned trajectory displayed on the same screen.

Table 1. Baseline characteristics of the patient population.

\begin{tabular}{llcl}
\hline & Age & Sex-ratio (M/F) & NVT \\
\hline CT Control group & $60 \pm 9$ & $12 / 4$ & $3[1 ; 3]$ \\
CAN group & $65 \pm 9$ & $13 / 8$ & $2[2 ; 3]$ \\
$P$-value & 0.079 & 0.05 & 0.087 \\
\hline
\end{tabular}

February 2017. Twenty-one patients (8women, 13 men; average age $65 \pm 9$ years) underwent vertebroplasty with CAN system (CAN group): the median number of vertebrae treated per patient was $2[2 ; 3]$. The other 16 patients ( 4 women, 12 men; average age $60 \pm 9$ years) had been subjected to the conventional percutaneous vertebroplasty procedure (CT control group)with median of $3[1 ; 3]$ vertebrae treated per patient.

Table 1 presents the mean values and standard deviations for patient age, and median number of vertebrae treated per patient (NVT) in the same session with the 25 and 75 percentiles.

A Fisher exact test was used to assess the difference in the number of vertebrae per patient treated between the two groups and showed no statistically significant difference $(p=0.087)$.

The IDLP, DLPT and procedure time were continuous variables that did not follow the normal distribution pattern (Shapiro-Wilk normality test, $p<0.05$ ).

\subsection{Main outcome}

\subsubsection{Dose radiation during the needle insertion phase}

The median IDLP in the CAN Group was 305.6 mGy.cm [182.3; 565.4] vs. 975.2 mGy.cm [568.3; 1077.075] in the CT Control Group $(p<0.001)$ (Fig. 2), i.e., a reduction by a 3.2 factor of the dose.

Since the number of vertebrae treated per patient (NVT) was lower in CAN group in comparison to the Control Group (although not statistically significant), the IDPL pervertebrae per treated patient were compared. The median IDLP per vertebra were $153 \mathrm{mGy} . \mathrm{cm}$ [106-213] in the CAN Group vs. 353 mGy.cm [305-445] in the Control Group. The reduction by a factor of 2.3 is statistically significant $(p<0.001)$ (Fig. 3).

\subsection{Secondary endpoints}

\subsubsection{Dose radiation during the total procedure}

Regarding the DLP during the total duration of the intervention (DLPT), the median was lower in the CAN Group than in the CT group (Tab. 2). This DLPT reduction in the CAN Group was also observed when comparing DLPT per vertebrae per patient treated between the two groups. Although this seemed to be a clinically meaningful reduction, it did not reach statistical significance (Tab. 2). 


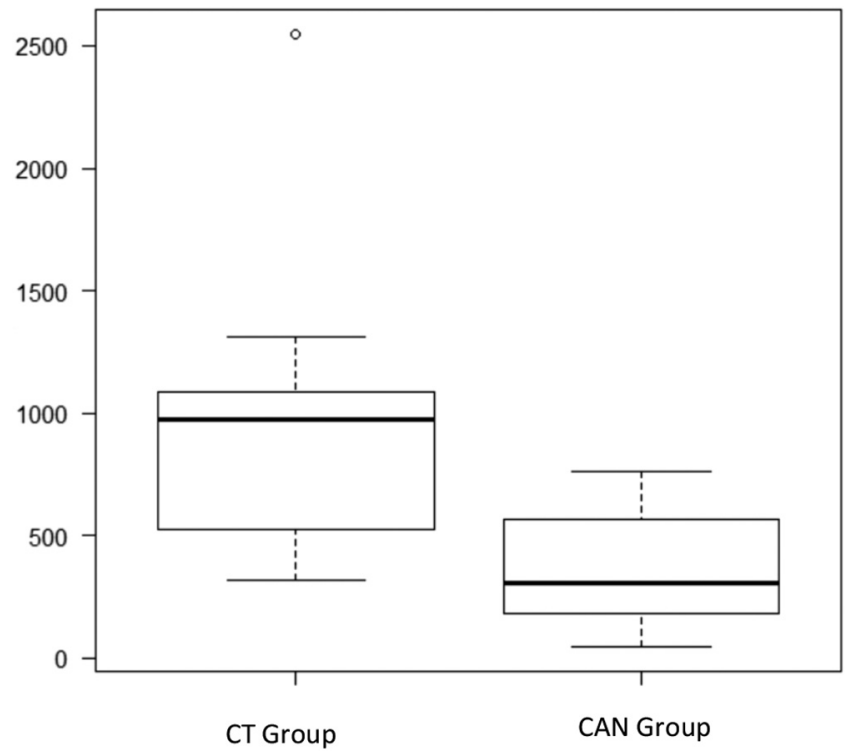

Fig. 2. Median IDLP (mGy.cm) per patient during the needle insertion phase (one outlier to be noticed in the control group).

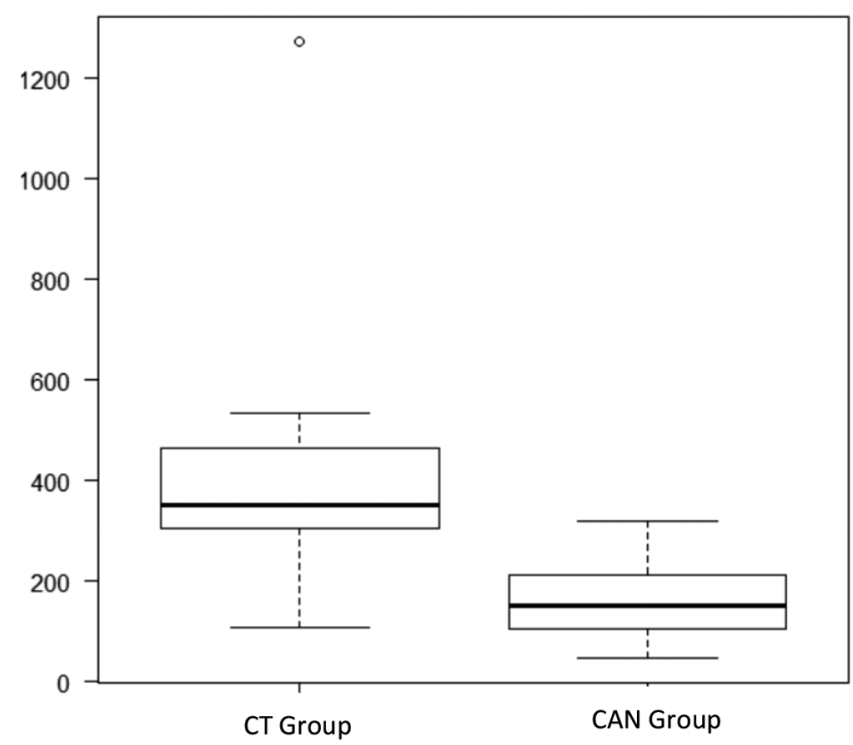

Fig. 3. Median IDLP (mGy.cm) per-vertebra per patient treated during the needle insertion phase.

\subsubsection{Procedure duration}

The median procedure duration in the CAN Group was $50 \mathrm{~min}[35 ; 60]$ vs. $100 \mathrm{~min}[100 ; 82](p<0.001)$ in the CT control group. This represented a $50 \%$ reduction in procedure duration (Fig. 4).

The reduction in the procedure duration in the CAN group remained significant when comparing the procedure duration per-vertebrae treated between the two groups: $20 \min [17 ; 23]$ vs. $39 \min [27 ; 51] ;(p<0.001)$.

\subsubsection{Complications}

No major complications were observed in the two groups.
Table 2. Median dose length product during the procedure.

\begin{tabular}{llll}
\hline & CAN group & CT group & p-values \\
\hline $\begin{array}{l}\text { DLPT (mGy.cm) } \\
\text { DLPT/NVT }\end{array}$ & $1883[1222 ; 2537]$ & $2116[1656 ; 3082]$ & 0.263 \\
(mGy.cm/vertebra) & $812[627 ; 1081]$ & $1090[639 ; 1815]$ & 0.495 \\
\hline
\end{tabular}

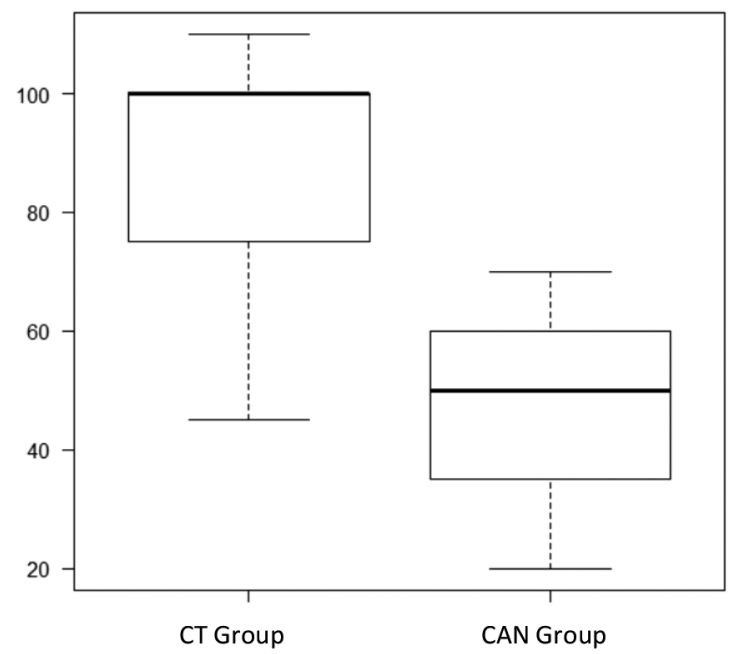

Fig. 4. Comparison of procedure duration ( $\mathrm{min}$ ) between CT group and CAN group.

\section{Discussion}

The prevalence of spine-related pathology has recently increased (Tarantino et al., 2007; Griffith and Guglielmi, 2010), due to increased life expectancy for cancer patients globally. In fact, up to $40 \%$ of patients with cancer develop spinal metastases, which often lead to vertebral compression fracture (Klimo and Schmidt, 2004). In addition, 20\% of the elderly population develop an osteoporotic vertebral fracture (Griffith and Guglielmi, 2010). This has led toward an increasing number of CT-guided minimally invasive procedures to reduce the potential complications and side effects among these patients (Boszczyk et al., 2006).

As a result, patients, interventional physicians, and operating room staff members are exposed to incrementally higher levels of radiation related to vertebral augmentation procedures. Reasonable concerns about interventional radiologists', surgeons' and patients' potential consequences have been raised (Li et al., 2013; Panizza et al., 2014). Several studies found that radiation-related risk during interventional procedures may be considerable (Harstall et al., 2005; Mastrangelo et al., 2005; Chou et al., 2010). The estimated cancer incidence among interventional physicians who perform image-guided percutaneous vertebroplasty was $0.025 \%$, and the estimated incidence of fatal thyroid cancer was $0.0025 \%$ (Harstall et al., 2005). This represents 25 times higher risk than the general population (Harstall et al., 2005). Interventional physician skin exposure during vertebroplasty procedure is up to $10 \%$ of the annual effective dose limit recommended by the International Commission on Radiological Protection 
(Harstall et al., 2005). The relative risk of lens opacity is $5.7(95 \%$ CI: 1.5-22) for interventional physicians (interventional cardiologist) compared to the general population (Ciraj-Bjelac et al., 2010). The relative risk of posterior subcapsular opacities is 3.2 among interventional physicians (interventional cardiologist) compared to the general population (Vano et al., 2010). The relative cancer risk of interventional orthopedic surgeons is 5.37 higher than the general population (Mastrangelo et al., 2005). The malignancies in the exposed personnel range from cancers of solid organs to skin and hematopoietic cancers (Mastrangelo et al., 2005). Consequently, there remains an unmet need for solutions to reduce the radiation dose exposure of both patients and interventional physicians, e.g., by reducing the duration of each exposure. In this study, the impact of a CAN system (CT-Navigation ${ }^{\mathrm{TM}}$ system by IMACTIS ${ }^{\circledR}$, France) on patient radiation dose exposure during percutaneous vertebroplasty was assessed.

A significant reduction of IDLP by a factor of 3.2 during the preparation of percutaneous vertebroplasty procedures was observed when using the CAN system. The IDLP represents the dose length product (DLP) during the needle insertion phase. Although the DLP does not take the size of the subject into account and is not a measure of absorbed dose, it is directly proportional to the effective dose (Huda et al., 2008). This reduction of radiation exposure could be mainly explained by the electromagnetic guidance provided by the system. Using the needle holder, the interventional radiologist obtained the optimal angle of approach. During needle advancement, no CT-controls were taken to detect the insertion depth. Only one CT control image was taken before the insertion of the needle in the vertebrae to ensure that the needle tip was in the planned trajectory.

Durand et al. in their prospective controlled trial found that there was no significant difference in patient radiation dose when using the same IMACTIS navigation system to perform interventional procedures (Durand et al., 2017). This result could be explained by the type of procedure carried out. They focused mainly on biopsy, ablation or infiltration, whereas, in our study we carried out only vertebroplasty procedures that require more radiation (Lonjon et al., 2016).

The DLPT was lower in the CAN Group than in the CT Group. This DLPT reduction in the CAN group of this study was also observed when comparing the DLPT per vertebrae per patient treated between the two groups. Although this appeared to be a clinically meaningful reduction, it did not reach statistical significance, most probably due to underpowering. This finding highlights the role of navigation systems in reducing radiation exposure and prevent related risks during certain higher risk interventional radiological procedures (Barzilay et al., 2014).

The procedure duration in the CAN group of this study was half that of the CT Control group. This statistically significant reduction in the vertebroplasty procedure time using a navigation system different from that of IMACTIS was also described in several studies (Perisinakis et al., 2004; Izadpanah et al., 2009; Barzilay et al., 2014). Conversely, Durand et al., found that the duration of the step from planning to initial needle, ground placement was shorter in the conventional procedure group than in the navigation system assisted group (Durand et al., 2017). This difference could be explained by the relevant learning curve associated with the use of the navigation systems, the operator experience and the type of procedure performed. In their study, the median number of interventions per operator was only 3 , whereas, in our study, one operator conducted all interventions (twenty-one interventions). Moreover, we carried out only vertebroplasty procedures, whereas they focused mainly on biopsy, ablation and infiltration (Durand et al., 2017).

As detailed above the CAN system assessed in this study significantly reduced both the need for radiation exposure and procedure duration, compared to the conventional CT guided percutaneous vertebroplasty procedure. However, there remain some limitations to this study. First, the study was a retrospective and non-randomized study and as such, subject to selection bias. Yet, the patients were consecutively recruited, which may reduce the impact of such bias. Second, the single-center singleradiologist setup, and the low study power, as well as the retrospective collection of data limits generalization of our findings. Hence, a larger properly powered prospective randomized control multi-center trial which could be appropriately powered based on these findings is needed to confirm the impact of the IMACTIS CAN system (CT-Navigation) on radiation dose exposure and procedure duration in percutaneous vertebroplasty.

\section{Conclusion}

In conclusion, this preliminary retrospective study demonstrated that the IMACTIS ${ }^{\circledR}$ CAN (CT-Navigation) system fulfilled an unmet need and enabled the interventional radiologist to reduce both patient radiation dose and procedure duration during percutaneous vertebroplasty. One can expect that interventional physician dose is reduced as well through the diminution of total exposure time although it was not monitored in this preliminary study.

\section{References}

Barzilay Y, Schroeder JE, Hiller N, Singer G, Hasharoni A, Safran O, Liebergall M, Itshayek E, Kaplan L. 2014. Robot-assisted vertebral body augmentation: A radiation reduction tool. Spine (Phila Pa 1976) 39: 153-157.

Bernier M-O, Jacob S, Maccia C, Bar O, Catelinois O, Blanchard D, Laurier D. 2012. Patient cumulative radiation exposure in interventional cardiology. Radioprotection 47: 93-103.

Boszczyk BM, Bierschneider M, Panzer S, Panzer W, Harstall R, Schmid K, Jaksche H. 2006. Fluoroscopic radiation exposure of the kyphoplasty patient. Eur. Spine J. 15: 347-355.

Bourguignon M, Bérard P, Bertho J, Farah J, Mercat C. 2017. What's next in Radioprotection? Radioprotection 52: 21-28.

Chou LB, Cox CA, Tung JJ, Harris AH, Brooks-Terrell D, Sieh W. 2010. Prevalence of cancer in female orthopaedic surgeons in the United States. J. Bone Joint Surg. Am. Vol. 92: 240-244.

Ciraj-Bjelac O, Rehani MM, Sim KH, Liew HB, Vano E, Kleiman NJ. 2010. Risk for radiation-induced cataract for staff in interventional cardiology: Is there reason for concern? Catheter. Cardiovasc. Interv. (official journal of the Soc. Card. Angiogr. Interv.) 76: 826-834.

Durand P, Moreau-Gaudry A, Silvent AS, Frandon J, Chipon E, Medici M, Bricault I. 2017. Computer assisted electromagnetic 
navigation improves accuracy in computed tomography guided interventions: A prospective randomized clinical trial. PloS one 12: e0173751.

Feghali J, Tagnard-Mérat F, Donadille L, Rehel J, Aubert B, Allodji R, Gauron C, Farah J, Clairand I. 2014. Statistical analysis of measured operators' finger doses in interventional radiology. Radioprotection 49: 91-99.

Friedly J, Standaert C, Chan L. 2010. Epidemiology of spine care: The back pain dilemma. Phys. Med. Rehabil. Clin. 21: 659-677.

Griffith JF, Guglielmi G. 2010. Vertebral fracture. Radiol. Clin. 48: 519-529.

Harstall R, Heini PF, Mini RL, Orler R. 2005. Radiation exposure to the surgeon during fluoroscopically assisted percutaneous vertebroplasty: A prospective study. Spine (Phila Pa 1976) 30: 1893-1898.

Huda W, Ogden KM, Khorasani MR. 2008. Converting doselength product to effective dose at CT. Radiology 248: 995-1003.

Izadpanah K, Konrad G, Südkamp NP, Oberst M. 2009. Computer navigation in balloon kyphoplasty reduces the intraoperative radiation exposure. Spine 34: 1325-1329.

Jacob S, Bar O, Catelinois O, Maccia C, Laurier D, Bernier M-O. 2013. Risk assessment for potential radiation-induced cancer after lung and bone marrow exposure during interventional cardiology procedures. Radioprotection 48: 203-213.

Klimo P, Schmidt MH. 2004. Surgical management of spinal metastases. Oncol. 9: 188-196.

Komemushi A, Tanigawa N, Kariya S, Kojima H, Shomura Y, Sawada S. 2005. Radiation exposure to operators during vertebroplasty. J. Vasc. Interv. Radiol. 16: 1327-1332.

Li Y-Y, Huang T-J, Cheng C-C, Wu M-H, Lee C-Y. 2013. Comparing radiation exposure during percutaneous vertebroplasty using onevs. two-fluoroscopic technique. BMC Musculoskelet. Disord. 14: 38.
Lonjon N, Le Corre M, Le Roy J, Greffier J, Fuentes S, Tonetti J, Charles YP, Blondel B, Kouyoumdjian P. 2016. Surgeon's and patient's radiation exposure through vertebral body cement augmentation procedures: A prospective multicentric study of 49 cases. World Neurosurg. 93: 371-376.

Mastrangelo G, Fedeli U, Fadda E, Giovanazzi A, Scoizzato L, Saia B. 2005. Increased cancer risk among surgeons in an orthopaedic hospital. Occup. Med. (Oxford, England) 55: 498-500.

Narain AS, Hijji FY, Yom KH, Kudaravalli KT, Haws BE, Singh K. 2017. Radiation exposure and reduction in the operating room: Perspectives and future directions in spine surgery. World J. Orthop. 8: 524.

Panizza D, Barbieri M, Parisoli F, Moro L. 2013. Patient radiation exposure during different kyphoplasty techniques. Radiat. Prot. Dosim. 158: 230-234.

Panizza D, Barbieri M, Parisoli F, Moro L. 2014. Patient radiation exposure during different kyphoplasty techniques. Radiat. Prot. Dosim. 158: 230-234.

Perisinakis K, Damilakis J, Theocharopoulos N, Papadokostakis G, Hadjipavlou A, Gourtsoyiannis N. 2004. Patient exposure and associated radiation risks from fluoroscopically guided vertebroplasty or kyphoplasty. Radiology 232: 701-707.

Synowitz M, Kiwit J. 2006. Surgeon's radiation exposure during percutaneous vertebroplasty. J. Neurosurg.: Spine 4: 106-109.

Tarantino U, Cannata G, Lecce D, Celi M, Cerocchi I, Iundusi R. 2007. Incidence of fragility fractures. Aging Clin. Exp. Res. 19: 7-11.

Toma CD, Dominkus M, Nedelcu T, Abdolvahab F, Assadian O, Krepler P, Kotz R. 2007. Metastatic bone disease: A 36-year single centre trend-analysis of patients admitted to a tertiary orthopaedic surgical department. J. Surg. Oncol. 96: 404-410.

Vano E, Kleiman NJ, Duran A, Rehani MM, Echeverri D, Cabrera M. 2010. Radiation cataract risk in interventional cardiology personnel. Radiat. Res. 174: 490-495

Cite this article as: Teriitehau C, Rabeh H, Pessis E, Sénéchal Q, Besse F, Bravetti M. 2020. Reduction of patient radiation dose during percutaneous CT vertebroplasty: Impact of a new computer-assisted navigation (CAN) system. Radioprotection 55(1): 11-16 